\title{
Role of nuclear spin-orbit coupling on the constitution of the outer crust of a nonaccreting neutron star
}

\author{
N. Chamel ${ }^{1, a}$, A. F. Fantina ${ }^{1,2}$, J. M. Pearson ${ }^{3}$, and S. Goriely ${ }^{1}$ \\ ${ }^{1}$ Institut d'Astronomie et d'Astrophysique, CP-226, Université Libre de Bruxelles (ULB), 1050 Brussels, Bel- \\ gium \\ ${ }^{2}$ Grand Accélérateur National d'lons Lourds (GANIL), CEA/DRF - CNRS/IN2P3, Bvd Henri Becquerel, \\ 14076 Caen, France \\ ${ }^{3}$ Dépt. de Physique, Université de Montréal, Montréal (Québec), H3C 3J7, Canada
}

\begin{abstract}
The role of the nuclear spin-orbit coupling on the equilibrium composition and on the equation of state of the outer crust of a nonaccreting neutron star is studied by employing a series of three different nuclear mass models based on the self-consistent Hartree-Fock-Bogoliubov method.
\end{abstract}

\section{Introduction}

Born in the aftermath of gravitational core-collapse supernova explosions, neutron stars are the most compact stars in the Universe [1]. The outermost part of these stars is expected to be made of a bodycentered cubic crystal of iron whose density increases with depth. At density $\rho_{\text {eip }} \approx 2 \times 10^{4} \mathrm{~g} \mathrm{~cm}^{-3}$, the interatomic spacing becomes comparable with the atomic radius so that atoms are ionized although thermal effects are typically small (see, e.g., Ref. [1]). At densities $\rho \gg \rho_{\text {eip }}$, the crust thus consists of a dense crystallized plasma of nuclei and highly degenerate electrons. As the density reaches about $7 \times 10^{6} \mathrm{~g} \mathrm{~cm}^{-3}$, the interelectron spacing becomes comparable to the electron Compton wavelength therefore relativistic effects on electrons can no longer be ignored. With further compression, iron becomes unstable against electron captures and various other nuclear processes (see, e.g., Ref. [2]). The equilibrium composition of the crust is completely determined by experimental nuclear masses up to a density of about $6 \times 10^{10} \mathrm{~g} \mathrm{~cm}^{-3}$. Recourse must be made to nuclear models for the crustal layers beneath. With increasing depth, nuclei become progressively more neutron rich (see, e.g., Refs. [3-8] for recent calculations). At some density $\rho_{\text {drip }} \approx 4.4 \times 10^{11} \mathrm{~g} \mathrm{~cm}^{-3}$, neutrons "drip" out of nuclei thus delimiting the boundary between the outer and inner regions of the crust (see, e.g., Ref. [9] for a recent discussion). The outer crust composition is essential to evaluate the possible contribution of neutron stars to the galactic enrichment in the so-called $r$-process nuclei, whose origin remains one of the major mysteries in astrophysics (see, e.g. Ref. [10]). The constitution of the crust is also important for determining the size of the highest mountains, which can be a source of gravitational wave emission [11], and for modelling various other astrophysical phenomena (see, e.g., Ref. [12]).

\footnotetext{
ae-mail: nchamel@ulb.ac.be
} 
In this paper, we study the role of nuclear spin-orbit coupling on the structure and on the equation of state of the outer crust of a nonaccreting neutron star using the experimental atomic mass measurements from the 2012 Atomic Mass Evaluation [13] (AME) complemented by the Brussels-Montreal Hartree-Fock-Bogoliubov (HFB) atomic mass tables HFB-24 [14], HFB-28 and HFB-29 [15].

\section{Model of the outer crust of a neutron star}

The model we adopt here is essentially the same as the one described in Ref. [4]. We will therefore only review the main assumptions.

We consider matter at densities $\rho \gg \rho_{\text {eip }}$ and temperatures $T$ much below the crystallization temperature $T_{m}$. We assume that matter is in full thermodynamic equilibrium [16, 17], in which case only one kind of nuclei with proton number $Z$ and mass number $A$ is generally present at any given density $[18,19]$. The crystallization temperature $T_{m}$ is thus given by (see, e.g., Ref. [1])

$$
T_{m}=\frac{e^{2}}{a_{e} k_{\mathrm{B}} \Gamma_{m}} Z^{5 / 3},
$$

$e$ is the elementary electric charge, $a_{e}=\left(3 /\left(4 \pi n_{e}\right)\right)^{1 / 3}$ is the electron-sphere radius, $n_{e}$ is the electron number density, $k_{\mathrm{B}}$ is Boltzmann's constant, and $\Gamma_{m} \simeq 175$ is the Coulomb coupling parameter at melting. Nuclei are further supposed to be arranged on a body-centered cubic lattice [20]. Since inside the star the pressure has to vary continuously and since the baryon number is conserved, the suitable thermodynamic potential for determining the equilibrium composition of the crust is the Gibbs free energy per baryon [21]. The latter is calculated as in Ref. [4] except that the contribution arising from electron polarization effects is now calculated as discussed in Ref. [22].

The only microscopic inputs are nuclear masses. In Ref. [4], we made use of the experimental atomic mass measurements from the 2003 AME [23]. Here we use the more recent data from the 2012 AME [13]. For the mases that have not yet been measured, we have employed three different microscopic nuclear mass models, namely HFB-24 [14], HFB-28 and HFB-29 [15]. All these models are based on the self-consistent HFB method using generalized Skyrme effective interactions [24] supplemented with a microscopic contact pairing interaction [25]. These models differ in their treatment of the spin-orbit coupling. The spin-orbit coupling between nucleons $i$ and $j$ underlying the HFB-24 model is of the standard form

$$
v_{i j}^{\mathrm{so}}=\frac{\mathrm{i}}{\hbar^{2}} W_{0}\left(\boldsymbol{\sigma}_{\boldsymbol{i}}+\boldsymbol{\sigma}_{\boldsymbol{j}}\right) \cdot \boldsymbol{p}_{i j} \times \delta\left(\boldsymbol{r}_{i j}\right) \boldsymbol{p}_{i j}
$$

where $\boldsymbol{r}_{i j}=\boldsymbol{r}_{i}-\boldsymbol{r}_{j}, \boldsymbol{p}_{i j}=-\mathrm{i} \hbar\left(\boldsymbol{\nabla}_{i}-\boldsymbol{\nabla}_{j}\right) / 2$ is the relative momentum, $\boldsymbol{\sigma}_{\boldsymbol{i}}$ and $\boldsymbol{\sigma}_{\boldsymbol{j}}$ denote the Pauli spin matrices. The model HFB-28 includes a density-dependent term

$$
v_{i j}^{\mathrm{so}} \rightarrow v_{i j}^{\mathrm{so}}+\frac{\mathrm{i}}{\hbar^{2}} W_{1}\left(\boldsymbol{\sigma}_{\boldsymbol{i}}+\boldsymbol{\sigma}_{\boldsymbol{j}}\right) \cdot \boldsymbol{p}_{i j} \times\left(n_{q i}+n_{q j}\right)^{v} \delta\left(\boldsymbol{r}_{i j}\right) \boldsymbol{p}_{i j}
$$

where $q=n, p$ for neutrons and protons respectively, and $n_{q i}$ and $n_{q j}$ are the local nucleon number densities. The HFB-29 model was constructed from a generalized expression for the spin-orbit contribution to the energy density functional

$$
\mathcal{E}_{\mathrm{so}}=\frac{1}{2}\left[\boldsymbol{J} \cdot \boldsymbol{\nabla} n+\left(1+y_{w}\right) \sum_{q} \boldsymbol{J}_{\boldsymbol{q}} \cdot \boldsymbol{\nabla} n_{q}\right]
$$

where $\boldsymbol{J}=\boldsymbol{J}_{\boldsymbol{n}}+\boldsymbol{J}_{\boldsymbol{p}}$, and $\boldsymbol{J}_{\boldsymbol{q}}$ is the spin-current vector. The parameters of these models were primarily fitted to the 2353 measured masses of nuclei with $N, Z \geq 8$ given in the 2012 AME with a root mean 
square deviation below $0.6 \mathrm{MeV}$. These models were simultaneously constrained to reproduce various properties of infinite homogeneous nuclear matter, as inferred from experiments and from many-body calculations using realistic nucleon-nucleon potentials. These properties include ${ }^{1} S_{0}$ pairing gaps, the incompressibility of symmetric nuclear matter at saturation, effective masses, and the equation of state of pure neutron matter. The models HFB-28 and HFB-29 with a nonstandard spin-orbit coupling yield a slightly better fit to nuclear masses than the model HFB-24. A study of the model and parameter uncertainties affecting the HFB mass predictions can be found in Ref. [26].

\section{Results}

We have determined the constitution of the outer crust of a nonaccreting neutron star by minimising the Gibbs free energy per nucleon increasing the pressure from $P=9 \times 10^{-12} \mathrm{MeV} \mathrm{fm}^{-3}$ up to the onset of the neutron-drip transition at pressure $P=P_{\text {drip }}$, with a pressure step $\delta P=1.003 P$. We have employed the nuclear mass tables available on BRUSLIB [27].

As shown in table 1 and figure 1, the crustal composition is found to be sensitive to the form of the spin-orbit coupling, especially in the intermediate region at densities between $5 \times 10^{-5}$ and $8 \times 10^{-5} \mathrm{fm}^{-3}$. The uncertainties pertain to the following nuclides: ${ }^{79} \mathrm{Cu},{ }^{128} \mathrm{Pd}$ (predicted by model HFB-28 only), ${ }^{80} \mathrm{Ni},{ }^{126} \mathrm{Ru}$, and ${ }^{121} \mathrm{Y}$. For comparison, the corresponding mass excesses predicted by the different models are indicated in table 2. The deviations amount to about $0.9 \mathrm{MeV}$ at most. Quite remarkably, all three models predict the same sequence of equilibrium nuclides in the deepest layers of the outer crust. Moreover, the neutron-drip transition is found to occur at the same density $\bar{n}_{\text {drip }}=2.56 \times 10^{-3} \mathrm{fm}^{-3}$ and pressure $P_{\text {drip }}=4.86 \times 10^{-3} \mathrm{MeV} \mathrm{fm}^{-3}$. As shown in figure 2, the equation of state relating the pressure $P$ to the density $\bar{n}$ is essentially the same for all models. The most notable differences can be seen in the pressure plateaus marking the transition between adjacent crustal layers. In the limit of ultrarelativistic electrons, the threshold pressure is approximately given by [19]

$$
P_{1 \rightarrow 2} \approx \frac{\left(\mu_{e}^{1 \rightarrow 2}\right)^{4}}{12 \pi^{2}(\hbar c)^{3}}\left(1-\frac{4 C \alpha}{\left(3 \pi^{2}\right)^{1 / 3}} \frac{A_{1} Z_{2}^{5 / 3}-A_{2} Z_{1}^{5 / 3}}{A_{1} Z_{2}-A_{2} Z_{1}}\right),
$$

where $\alpha$ is the fine structure constant, $C=-1.44423$ [28], and the threshold electron Fermi energy is defined by

$$
\mu_{e}^{1 \rightarrow 2} \equiv\left[\frac{M^{\prime}\left(A_{2}, Z_{2}\right) c^{2}}{A_{2}}-\frac{M^{\prime}\left(A_{1}, Z_{1}\right) c^{2}}{A_{1}}\right]\left(\frac{Z_{1}}{A_{1}}-\frac{Z_{2}}{A_{2}}\right)^{-1}+m_{e} c^{2},
$$

in terms of the nuclear masses $M^{\prime}\left(A_{1}, Z_{1}\right)$ and $M^{\prime}\left(A_{2}, Z_{2}\right)$, and the electron mass $m_{e}$. The associated density discontinuity is approximately given by

$$
\frac{n_{2}^{\min }-n_{1}^{\max }}{n_{1}^{\max }} \approx \frac{A_{2}}{Z_{2}} \frac{Z_{1}}{A_{1}}\left[1+\frac{C \alpha}{\left(3 \pi^{2}\right)^{1 / 3}}\left(Z_{1}^{2 / 3}-Z_{2}^{2 / 3}\right)\right]-1 .
$$

\section{Conclusion}

We have studied the role of the nuclear spin-orbit coupling on the equilibrium composition and on the equation of state of the outer crust of a nonaccreting neutron star. To this end, we have minimised the Gibbs free energy per nucleon at fixed pressures, making use of experimental atomic masses from the 2012 AME [13] complemented by three different nuclear mass models HFB-24 [14], HFB-28 and 
Table 1. Sequence of equilibrium nuclides with increasing depth in the outer crust of a nonaccreting neutron star for different nuclear mass models. The nuclides with experimentally measured masses are indicated in boldface.

\begin{tabular}{ccc}
\hline HFB-28 & HFB-29 & HFB-24 \\
\hline${ }^{56} \mathbf{F e}$ & ${ }^{56} \mathbf{F e}$ & ${ }^{56} \mathbf{F e}$ \\
${ }^{62} \mathbf{N i}$ & ${ }^{62} \mathbf{N i}$ & ${ }^{62} \mathbf{N i}$ \\
${ }^{64} \mathbf{N i}$ & ${ }^{64} \mathbf{N i}$ & ${ }^{64} \mathbf{N i}$ \\
${ }^{66} \mathbf{N i}$ & ${ }^{66} \mathbf{N i}$ & ${ }^{66} \mathbf{N i}$ \\
${ }^{86} \mathbf{K r}$ & ${ }^{86} \mathbf{K r}$ & ${ }^{86} \mathbf{K r}$ \\
${ }^{84} \mathbf{S e}$ & ${ }^{84} \mathbf{S e}$ & ${ }^{84} \mathbf{S e}$ \\
${ }^{82} \mathbf{G e}$ & ${ }^{82} \mathbf{G e}$ & ${ }^{82} \mathbf{G e}$ \\
${ }^{80} \mathbf{Z n}$ & ${ }^{80} \mathbf{Z n}$ & ${ }^{80} \mathbf{Z n}$ \\
${ }^{79} \mathrm{Cu}$ & ${ }^{79} \mathrm{Cu}$ & - \\
${ }^{78} \mathrm{Ni}$ & ${ }^{78} \mathrm{Ni}$ & ${ }^{78} \mathrm{Ni}$ \\
${ }^{128} \mathrm{Pd}$ & - & - \\
${ }^{80} \mathrm{Ni}$ & - & ${ }^{80} \mathrm{Ni}$ \\
${ }^{126} \mathrm{Ru}$ & ${ }^{126} \mathrm{Ru}$ & - \\
${ }^{124} \mathrm{Mo}$ & ${ }^{124} \mathrm{Mo}$ & ${ }^{124} \mathrm{Mo}$ \\
${ }^{122} \mathrm{Zr}$ & ${ }^{122} \mathrm{Zr}$ & ${ }^{122} \mathrm{Zr}$ \\
- & ${ }^{121} \mathrm{Y}$ & ${ }^{121} \mathrm{Y}$ \\
${ }^{120} \mathrm{Sr}$ & ${ }^{120} \mathrm{Sr}$ & ${ }^{120} \mathrm{Sr}$ \\
${ }^{122} \mathrm{Sr}$ & ${ }^{122} \mathrm{Sr}$ & ${ }^{122} \mathrm{Sr}$ \\
${ }^{124} \mathrm{Sr}$ & ${ }^{124} \mathrm{Sr}$ & ${ }^{124} \mathrm{Sr}$ \\
\hline
\end{tabular}

Table 2. Mass excess (in $\mathrm{MeV}$ ) for different nuclei found in the outer crust of a neutron star, as predicted by different nuclear mass models.

\begin{tabular}{cccc}
\hline & HFB-28 & HFB-29 & HFB-24 \\
\hline${ }^{79} \mathrm{Cu}$ & -42.72 & -42.78 & -43.0 \\
${ }^{128} \mathrm{Pd}$ & -45.98 & -46.03 & -45.82 \\
${ }^{80} \mathrm{Ni}$ & -23.30 & -23.34 & -23.58 \\
${ }^{126} \mathrm{Ru}$ & -26.28 & -26.96 & -26.08 \\
${ }^{121} \mathrm{Y}$ & 32.57 & 32.26 & 32.99 \\
\hline
\end{tabular}

HFB-29 [15]. All these models yield excellent fits to all measured masses of nuclei with $Z, N \geq 8$ with a root-mean-square error below $0.6 \mathrm{MeV}$, but are based on different forms of the nuclear spin-orbit coupling. Although the equations of state predicted by these models are almost undistinguishable, differences have been found on the composition at densities between $5 \times 10^{-5}$ and $8 \times 10^{-5} \mathrm{fm}^{-3}$. A better understanding of the nuclear structure of very exotic nuclei (especially ${ }^{79} \mathrm{Cu},{ }^{128} \mathrm{Pd},{ }^{80} \mathrm{Ni},{ }^{126} \mathrm{Ru}$, and ${ }^{121} \mathrm{Y}$ ) is needed to reliably determine the internal constitution of the outer crust of a nonaccreting neutron star.

This work was financially supported by Fonds de la Recherche Scientifique - FNRS (Belgium), NSERC (Canada) and the European Cooperation in Science and Technology (COST) Action MP1304 "NewCompStar". 

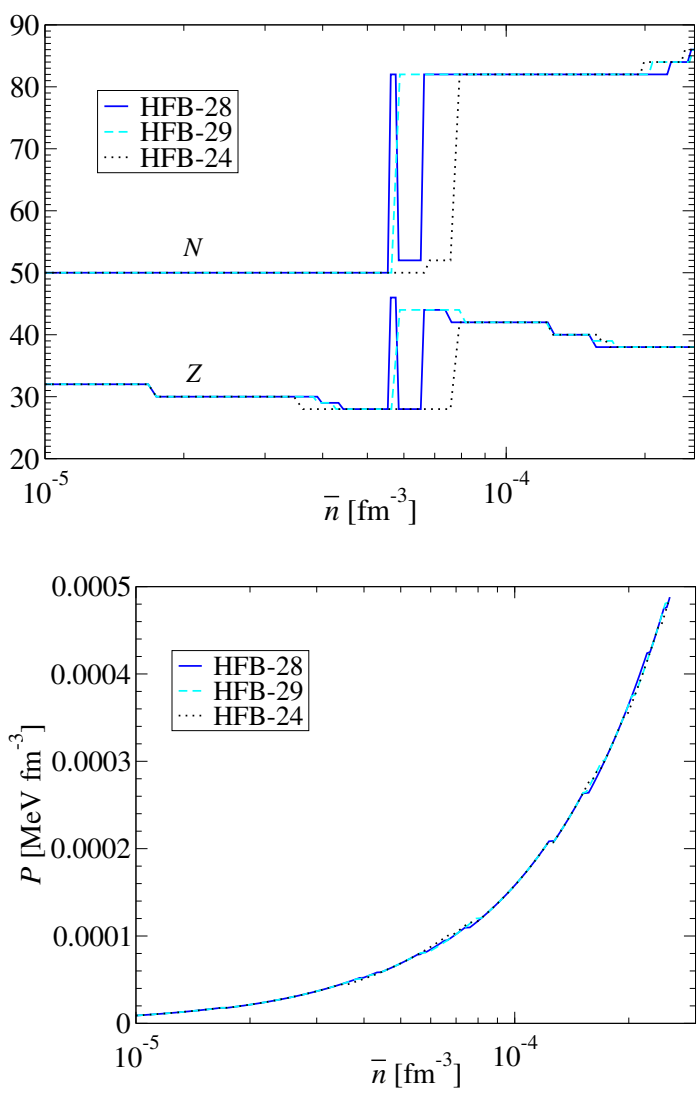

Figure 1. Proton number $Z$ and neutron number $N$ of equilibrium nuclei in the outer crust of a neutron star for different nuclear mass models as a function of the mean baryon number density $\bar{n}$.

Figure 2. Pressure $P$ vs mean baryon number density $\bar{n}$ in the outer crust of a neutron star for different nuclear mass models.

\section{References}

[1] P. Haensel, A. Y. Potekhin, and D. G. Yakovlev, Neutron Stars 1: Equation of state and structure (Springer, 2007).

[2] N. Chamel, A. F. Fantina, Phys. Rev. D 92, 023008 (2015).

[3] X. Roca-Maza and J. Piekarewicz, Phys. Rev. C 78, 025807 (2008).

[4] J. M. Pearson, S. Goriely, and N. Chamel, Phys. Rev. C 83, 065810 (2011).

[5] R. N. Wolf et al., Phys. Rev. Lett. 110, 041101 (2013).

[6] S. Kreim, M. Hempel, D. Lunney, and J. Schaffner-Bielich, Int. J. Mass Spec. 349-350, 63 (2013).

[7] N. Chamel, J. M. Pearson, A. F. Fantina, C. Ducoin, S. Goriely, and A. Pastore, Acta Phys. Pol. B 46, 349 (2015).

[8] R. Utama, J. Piekarewicz, and H. B. Prosper, Phys. Rev. C 93, 014311 (2016).

[9] N. Chamel, A. F. Fantina, J. L. Zdunik, P. Haensel, Phys. Rev. C 91, 055803 (2015).

[10] S. Goriely, N. Chamel, H.-T. Janka, J. M. Pearson, A\&A 531, A78 (2011).

[11] B. Haskell, D. I. Jones, N. Andersson, Mon. Not. R. Astron. Soc. 373, 1423 (2006).

[12] N. Chamel and P. Haensel, Living Rev. Relativity 11, 10 (2008); http://www.livingreviews.org/ lrr-2008-10

[13] G. Audi, M. Wang, A. H. Wapstra, F. G. Kondev, M. MacCormick, X. Xu, B. Pfeiffer, Chin. Phys. C 36, 1287 (2012). 
[14] S. Goriely, N. Chamel, J. M. Pearson, Phys. Rev. C 88, 024308 (2013).

[15] S. Goriely, Nuclear Physics A 933, 68 (2015).

[16] B. K. Harrison, and J. A. Wheeler, in Onzième Conseil de Physique Solvay (Stoops, Bruxelles, Belgium, 1958).

[17] B. K. Harrison, K. S. Thorne, M. Wakano, and J. A. Wheeler, Gravitation Theory and Gravitational Collapse (The University of Chicago Press, 1965).

[18] C. J. Jog and R. A. Smith, Astrophys. J.253, 839 (1982).

[19] N. Chamel, A. F. Fantina, Phys. Rev. C in press.

[20] M. Ruderman, Nature 218, 1128 (1968).

[21] G. Baym, C. Pethick, and P. Sutherland, Ap. J. 170, 299 (1971).

[22] N. Chamel, A. F. Fantina, Phys. Rev. D 93, 063001 (2016).

[23] G. Audi, A.H. Wapstra, and C. Thibault, Nucl. Phys. A729, 337 (2003).

[24] N. Chamel, S. Goriely and J. M. Pearson, Phys. Rev. C 80,065804 (2009).

[25] N. Chamel, Phys. Rev. C 82, 014313 (2010).

[26] S. Goriely and R. Capote, Phys. Rev. C 89, 054318 (2014).

[27] http://www.astro.ulb.ac.be/bruslib.

[28] R.A. Coldwell-Horsfall and A.A. Maradudin, J.Math.Phys. 1, 395 (1960). 